British Journal of Psychiatry (1996), 168, 127-129

\title{
Correspondence
}

Contents: Psychological treatments for depression/ Risperidone in schizophrenia/The essence of supportive psychotherapy/Biological psychiatry and psychotherapy.

\section{Psychological treatments for depression}

SIR: We offer three comments on Professor Scott's timely review of psychological treatments for depression (Scott, 1995).

First, the criteria for successful "manualisation" of a treatment have not been specified sufficiently thoroughly to guide clinical practice. The term is generally taken to mean that there are agreed procedures to which a trained therapist will adhere to a criterion level. However current manuals tend to specify criteria to be achieved in basic training but do not describe the more complex choices and decisions made in actual practice.

Second, the fourfold classification into behavioural, cognitive, interpersonal and dynamic therapies is unrepresentative of research and practice in the UK. Therapeutic practice is less narrowly channelled than this implies. Cognitive analytic therapy integrates strategies from cognitive and dynamic traditions, rather than residing solely within the dynamic class to which Professor Scott assigns it. Many practitioners combine cognitive and behavioural approaches in their daily practice; similarly, others combine dynamic and interpersonal strategies. How treatments are best categorised is an empirical question. For example, the finding by Jacobson et al (in press) that the behavioural activation component of Beck's cognitive therapy was as effective as the full treatment argues against CT's distinctiveness from the larger family of cognitive-behavioural methods.

The research-practice gap is therefore appropriately bridged via somewhat broader treatment protocols such as cognitive-behavioural (CB) and psychodynamic-interpersonal (PI) methods (Shapiro, 1995). Two comparative trials (e.g. Shapiro et al, 1994) established similar efficacy of manualised CB and PI treatments, and showed that therapists can adhere consistently to these models, even in the demanding context of research requiring them to practise both. For the PI method of these studies (Hobson's Conversational Model) there are well established and effective training methods (Margison \& Moss, 1994).

Finally, we are concerned that research on "pure" depression does not correspond to practice in which depression often co-exists with personality disorder, anxiety disorder, substance abuse and other co-morbid conditions. When assessing overall effectiveness in practice, as contrasted with efficacy as established in research trials, the impact of this comorbidity needs to be taken into account.

Jacobson N. S., Dobson, K. A., Truax, P. A., el al (in press) A component analysis of cognitive-behavioural treatment for depression. Journal of Consulting and Clinical Psychology.

MARGson F. \& Moss, S. (1994) Teaching psychotherapy skills to inexperienced psychiatry trainees using the Conversational Model. Psychotherapy Research, 4, 141-148.

ScotT, J. (1995) Psychological treatments for depression: An update. British Journal of Psychiatry, 167, 289-292.

Shaptro, D. A. (1995) Finding out how psychotherapies help people change. Psychotherapy Research, 5, 1-21.

-. BARKhin, M., Rees, A., et al (1994) Effects of treatment duration and severity of depression on the effectiveness of cognitive-behavioural and psychodynamic-interpersonal psychotherapy. Journal of Consulting and Clinical Psychology, 62 $522-534$.

Manchester Infirmary

F. MARgISON

Psychological Therapies Research Centre

D. A. Shapiro University of Leeds LS2 9JT

SIR: In her excellent review of this subject (Scott, 1995), Professor Scott says that the therapist's level of skill and adherence to the treatment model account for up to $30 \%$ of the variance in outcome. In that case, it seems to become very difficult to make group comparisons between these therapies and others, or indeed to make any general statement on the efficacy of psychological methods.

In fact, the data relating to psychological treatments in depression have come very largely from highly specialised centres, often associated with 